\title{
Sustainability of Ophthalmology Practice and Training During and Post the Pandemic of Coronavirus (COVID-19): A Review
}

\author{
Ahmed Almazroa ${ }^{1,2}$ \\ 'Imaging Research Department, King \\ Abdullah International Medical Research \\ Center, Riyadh, Saudi Arabia; ${ }^{2}$ King Saud \\ Bin Abdulaziz University for Health \\ Sciences, Riyadh, Saudi Arabia
}

Purpose: To review and analyse the globally established ophthalmic practice protocols during the coronavirus disease (COVID-19).

Methods: A literature review using search strategy was conducted to identify appropriate publications relevant to COVID-19 and ophthalmology practice and training. The safety and feasibility of the protocols were illustrated and discussed.

Results: Challenges in different eye care settings at various international ophthalmology departments have identified and analysed to introduce solutions. Several clinical protocols were established and concerned for screening procedures, waiting area, clinical flow (ie, patients' registration, personal (patients and healthcare workers) protection), and equipment safety in the clinics and operation rooms.

Discussion: In the review of this protocol, the strategic and operational missions of the Academic Medical Centers (AMCs) are demonstrated and discussed. This is in addition to the sustainability of the established protocols for cataract surgeries and glaucoma clinics and training during and after COVID-19.

Conclusion: All the protocols have established for temporary circumstances, such as postponing elective appointments and surgeries as well as applying the technology for regular follow-ups (transmission of image, video, and face-to-face interactions via widely available applications). Only, one protocol was stronger for the sustainability. Accordingly, recommendations are suggested for clinical sustainability during and after COVID-19.

Keywords: SARS-CoV-2, ophthalmic practice, healthcare workers, clinical management, infection control

\section{Introduction}

Affected patients and healthcare workers (HCWs) with various infectious diseases may frequently present at the eye clinics. Since they have direct contact with clinical equipment, it increases the risk of spreading COVID-19. Globally, the number of COVID-19 cases are still dramatically increasing. The HCWs are at high risk of being exposed to COVID-19. According to the Pan American Health Organization (PAHO), in September 2020, the COVID-19 has infected 570,000 HCWs and killed 2500 in the USA. ${ }^{1}$ In a recently published paper, Bandyopadhyay et $\mathrm{al}^{2}$ estimated the healthcare worker's (HCW's) COVID-19 infections and deaths. The analysis showed the case fatality rate for the HCWs ages between 18-29, 30-39, 40-49, 50-59, 60-69 and above 70-year old were 1.1\%,0.8\%, 1.1\%, 2.6\%, $7.1 \%$, and $37.2 \%$, respectively. Nguyen et $\mathrm{al}^{3}$ conducted a study (in the USA and
Correspondence: Ahmed Almazroa King Abdullah International Medical Research Center, King Saud Bin Abdulaziz University for Health Sciences, Ministry of National Guard, P.O. Box 3660, Riyadh,

I I 48I, Saudi Arabia

Tel +966II4294324

Email almazroaah@ngha.med.sa 
the UK) and found that the HCWs are at increased threat for reporting positive COVID-19 test results compared to the general community. The study has shown that the prevalence of COVID-19 for the HCWs was 2747 cases per 100,000 people and for the general community was 242 cases per 100,000 people.

During the COVID-19 pandemic, the HCWs are working for long hours in high-pressure circumstances. Additionally, they are at increased risk of infection; thus, they are also at a risk of infecting their families. Consequently, these causes are likely to increase the risk of mental health disorders. Greenberg ${ }^{4}$ has suggested six key elements to protect the mental health of the HCWs and are as follows:

proper acknowledgment of the hard work to foster resilience,

contacting the staff who does not turn up to work, in case his/her absence is due to impacted mental health,

the HCWs should receive the "return to normal work" interviews by expert mental health supervisors once COVID-19 begins to recede,

paying attention to HCWs in high-risk groups and/or being overwhelmed,

monitoring anyone who is at higher risk of developing mental health problems, and

managers should assist anyone who has been exposed to morally distressing circumstances.

As the number of COVID-19 positive cases increases, the transmission is also expected to increase. Unfortunately, there is a lack of well-established protocols dedicated to attenuate healthcare workers' risk. ${ }^{5}$ Thus, strict protocols are required for more protection at the clinic, taking into account the clinic's sustainability.

\section{Methods}

\section{Inclusion and Exclusion Criteria}

The analysis inclusion criteria concerned all the ophthalmology practice and training protocols during and after COVID19 for inpatients and outpatients. It is worthwhile to mention that the exclusion criteria was any ophthalmology practice and training protocols not dedicated to COVID-19.

\section{Research Criteria}

For this review, the reference articles were collected from January 2020 to January 2021 using standard web search engines, such as Google Scholar, PubMed, Web of Science. The keywords to search the literature were ophthalmology protocols during COVID-19, ophthalmology training during COVID-19, ocular surgeries during COVID-19, ophthalmology inpatients, and ophthalmology outpatient during COVID-19. Through the electronic databases, 483 citations were selected. Then, the unrelated titles were removed. A screening of abstracts was conducted for 69 citations. Full-text screening was performed for 13 manuscripts. Finally, six papers, comprising four original articles and two review papers fulfilled the inclusion criteria.

\section{Study Selection}

For this review, original articles and review papers regarding the ophthalmology practices and training protocols during COVID-19 were chosen.

\section{Ethical Consideration}

No ethical considerations were required for this review.

\section{Results}

Globally, the ophthalmology clinics have responded to COVID-19 in various ways to manage the risks to the staff and patients in the absence of respiratory symptoms. In this regard, different protocols have established (see Figures 1-6). Safadi et $\mathrm{al}^{6}$ have established a protocol, as shown in Figure 1, which covers the safety and effectiveness of hospital settings. Three subdivided categories for infection measures were considered in their practice protocol and are given below:

The use of personal protection (PPE), including wearing masks and eye protection. The patients are requested only to speak when asked during the slitlamp examination.

Environmental control for slit-lamp testing is undertaken by installing large protective plastic shields between the patient and the practitioner. Disinfection has to be performed, after each patient, on all clinical equipment and the air ventilation in the waiting areas.

Administrative control by first identifying the patients exposed to COVID-19 to follow the procedures indicated in Figure 1.

Other specific measures have contemplated, such as encouraging the use of telemedicine, dividing the workers into teams, separating the imaging areas, admitting only 


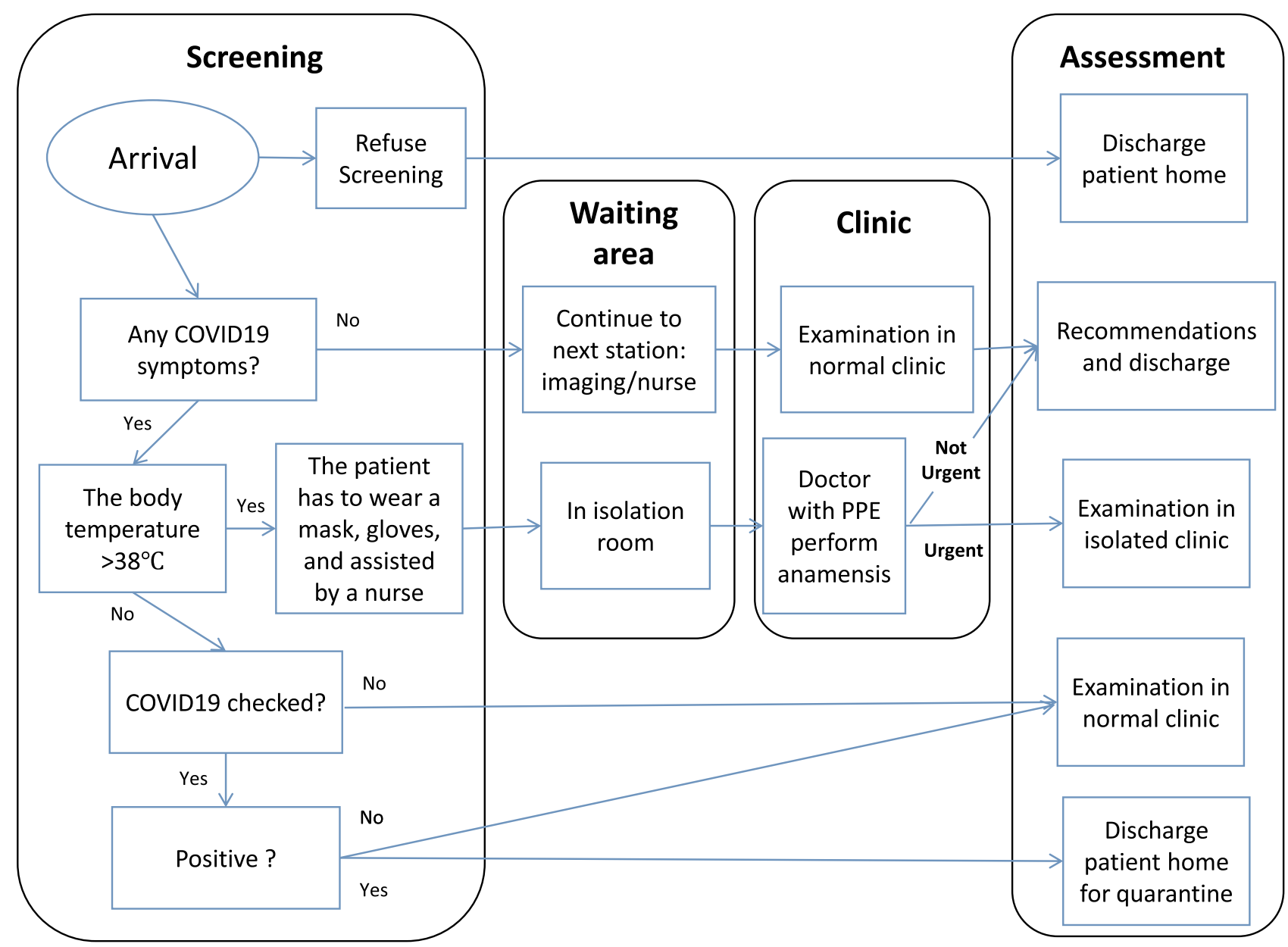

Figure I Flow chart of patients in ophthalmology clinics.

Note: Reproduced from Ophthalmology practice during the COVID-19 pandemic, Safadi K, Kruger JM, Chowers I, et al., 5, e000487, copyright 2020] with permission from BMJ Publishing Group Ltd. ${ }^{6}$

urgent cases for surgery, and check-ups for staff members. Optometry services were closed, except for biometry tests for urgent surgeries. The contact lenses fitting can raise concerns regarding coronavirus transmission, thus, increasing the risk of exposure to the virus.

Lim et $\mathrm{al}^{7}$ have stated the challenges affecting the ophthalmic practice in the ophthalmology department at Tan Tock Seng Hospital, the leading centre for managing COVID-19 patients in Singapore. The protocol is more comprehensive by covering both inpatient and outpatient care for the patients and their accompanying persons, as shown in Figure 2. Enhancing the detection of COVID-19 cases and minimising the transmission risks are some of the challenges healthcare workers face. Thus, guidelines for the ophthalmic practice were addressed, which included:

general infection control by the use of appropriate cleaning agents instead of the routine cleaning procedures, as well as washing hands with specific solutions after each patient,

outpatient care includes general and high-risk patients, where the aforementioned PPE is applied, and the consumable single-use equipment/materials should be used when treating high-risk patients,

inpatient care includes surgeries, where a proper PPE is worn and N95 mask is required for all operating staff, and

preparedness plans by continuously expanding the capacity to manage outbreak infectious diseases and logistic by maintaining a six-month stockpile of PPE and masks.

Sadhu et $\mathrm{al}^{8}$ have provided a review to discuss the evidence of detecting the COVID-19 from tears and proposed an approach implemented in the ophthalmic practice to protect healthcare workers and patients, as shown in 


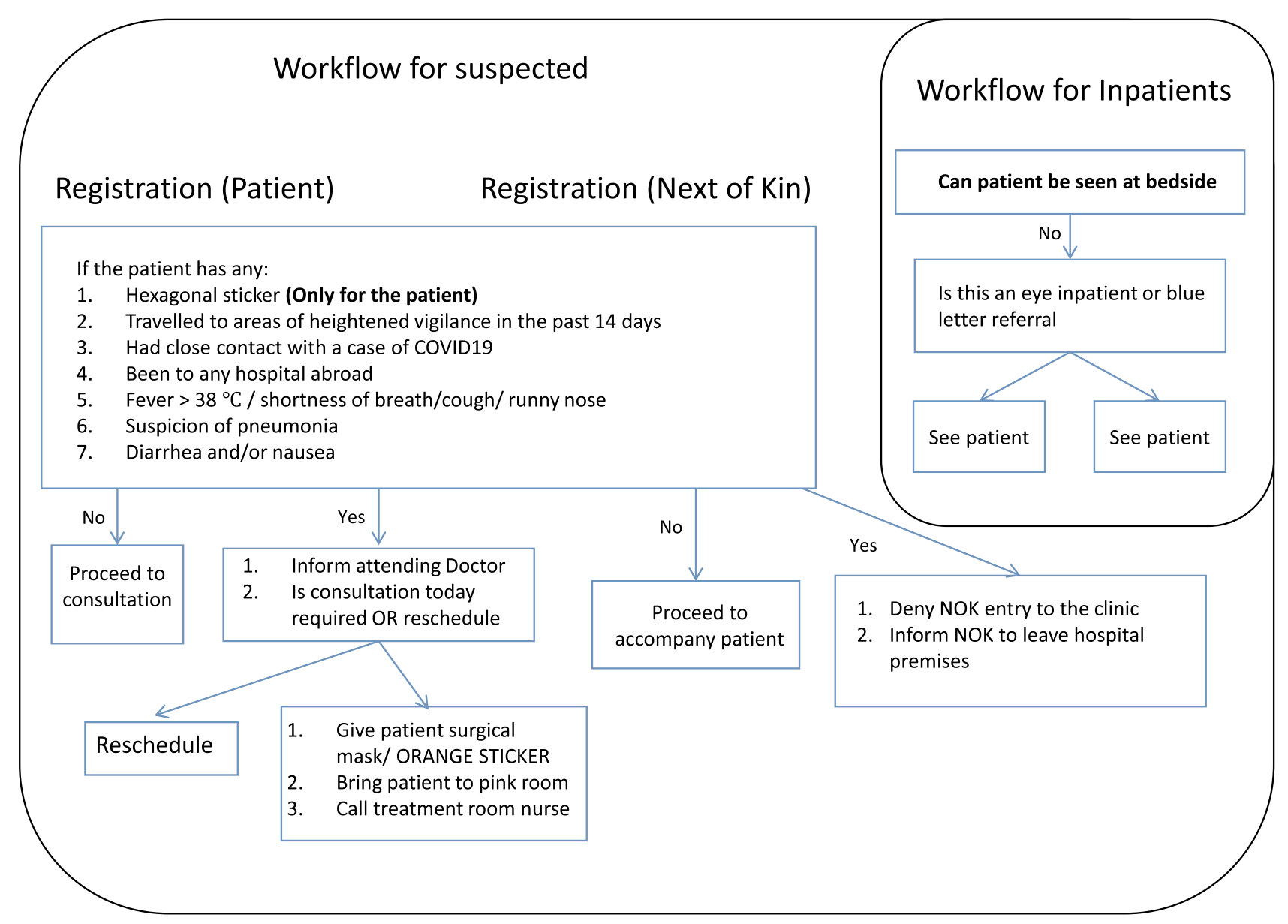

Figure 2 Registration workflow for outpatients and inpatient. Blue letter referral: inpatient interdisciplinary referrals to outpatients to be seen after 9:30 AM. Note: Reprinted by permission from Springer Nature Customer Service Centre GmbH: Springer Nature, Graefe's Archive for Clinical and Experimental Ophthalmology, Sustainable practice of ophthalmology during COVID-19: challenges and solutions, Lim LW, Yip LW, Tay HW, Ang XL, Lee LK. Copyright 2020. ${ }^{7}$

Figure 3. Minimising the exposures was the main target and was done by postponing all routine surgeries and elective appointments. The attendance is limited to emergency patients, and social distancing was strictly observed in the waiting areas. For suspicious COVID-19 patients, the PPE and surgical mask are crucial. Furthermore, the contact time was kept to a minimum, and slit-lamp shields were installed. In addition to that, for the necessary surgeries, sterilisation procedures were performed.

Gharebaghi et $\mathrm{al}^{9}$ have proposed procedures to put into place in the ophthalmic practice for suspected and emergency COVID-19 cases, as illustrated in Figure 4. The recommendations comprise the following:

Triage protocol to minimise patients' attendance in the clinic by conducting a screening protocol at the triage desk. Patients with low ophthalmologic acuity are postponed or referred to a telemedicine tool.
Necessity of PPE for all clinical staff, including face shields, gloves, water-resistance gowns, goggles, and respiratory protective equipment.

Regarding the workflow as some appointments cannot be postponed; thus, additional screenings were instituted, including taking patients' temperature to identify active fever. Also, social distancing represented by two metres was heeded.

Medical instrument disinfection is highly considered, and plastic shields were installed on the slit-lamp.

Surgical considerations comprise a screening test prior to the surgery and avoiding general anaesthesia as much as possible.

Moreover, Romano et $\mathrm{al}^{10}$ have presented guidelines to curtail virus infection in the ophthalmic clinics' outpatients. This is based on the emerging literature and their experience from hospitals in Bergamo, Italy. The triage 


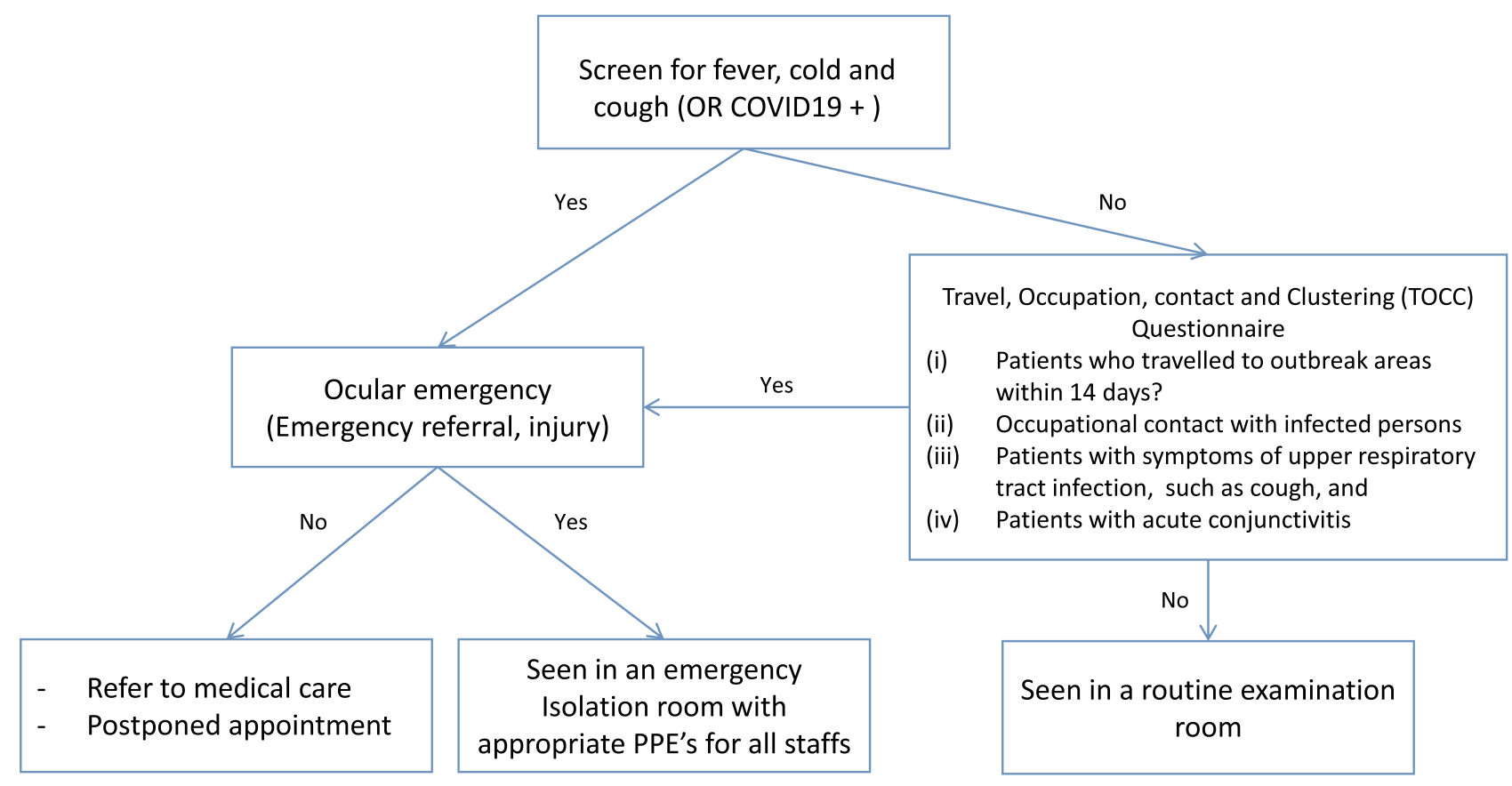

Figure 3 Flowchart of the patient triage protocol.

Note: Copied from COVID-19: limiting the risks for eye care professionals, Sadhu S, Agrawal R, Pyare R, et al., Ocular Immunology and Inflammation, 2020 Taylor \& Francis, reprinted by permission of the publisher (Taylor \&Francis Ltd, http://www.tandfonline.com). ${ }^{8}$

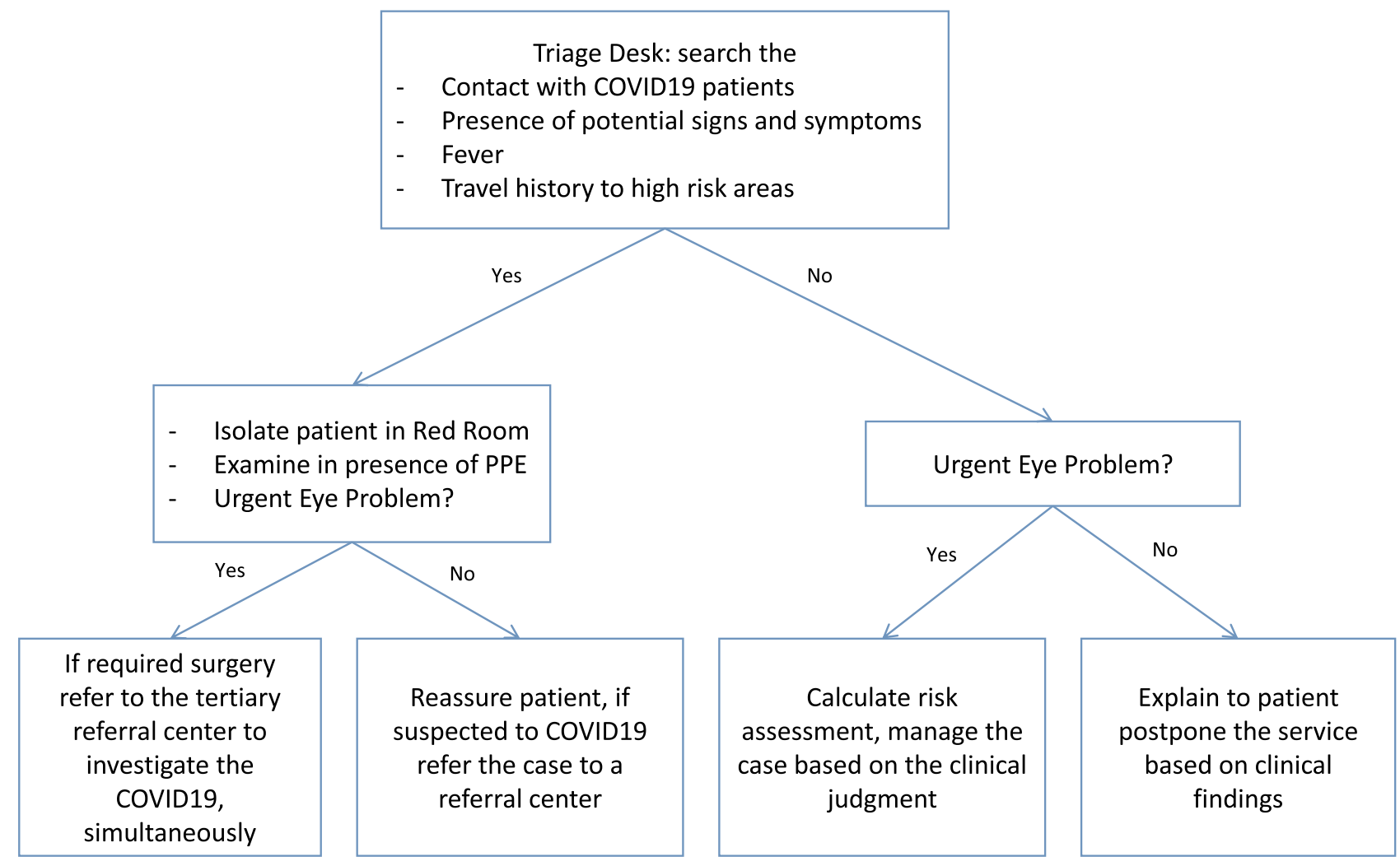

Figure 4 Decision tree in the eye clinic during the COVID-19 pandemic.

Note: Reproduced from Gharebaghi R, Desuatels J, Moshirfar M, Parvizi M, Daryabari SH, Heidary F. COVID-I9 preliminary clinical guidelines for ophthalmology practices. Med Hypothesis Discov Innov Ophthalmol. 2020;8(2):149.9 


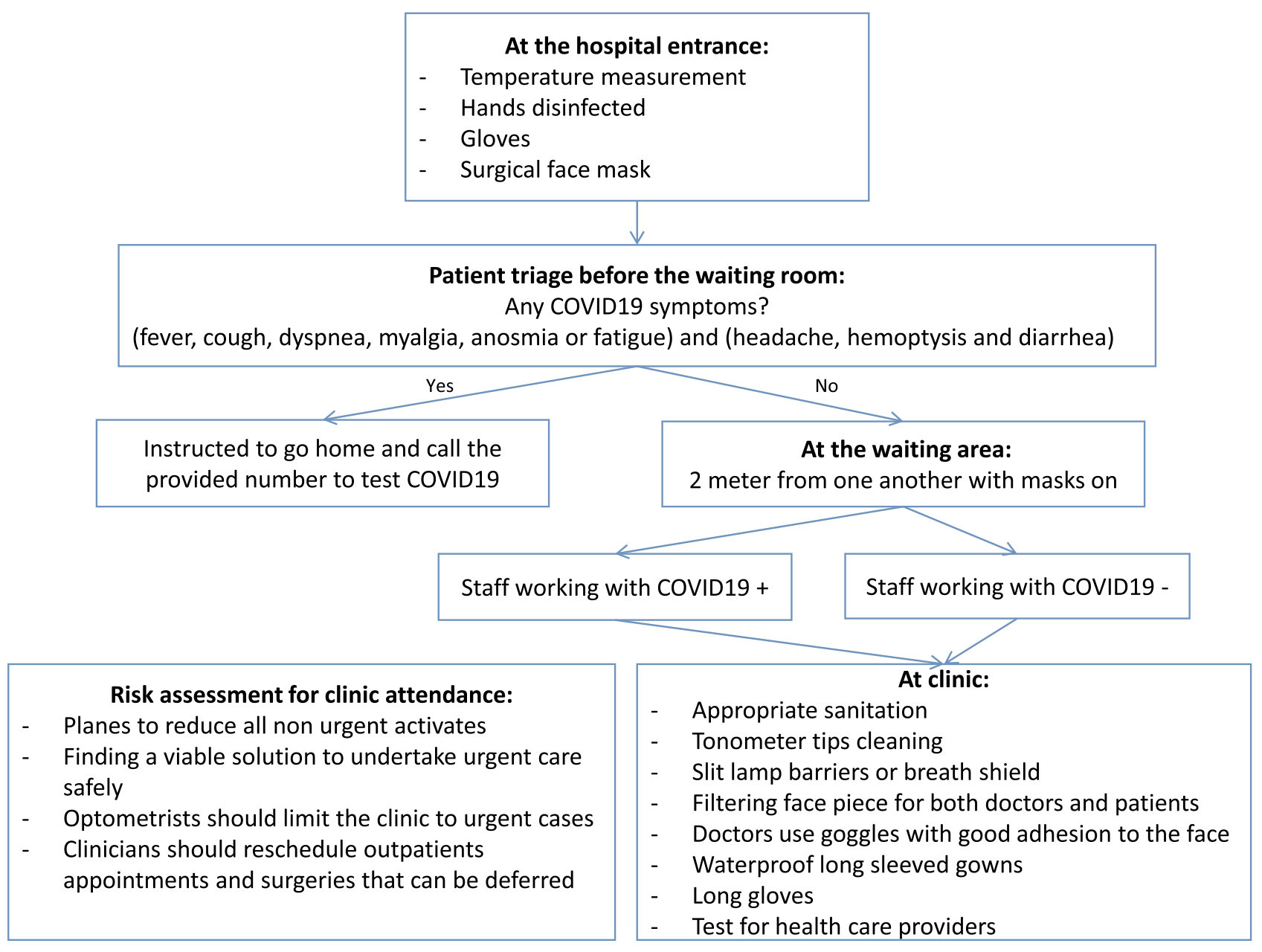

Figure 5 Decision tree in the ophthalmic outpatients' clinic during the COVID-19 pandemic.

Note: Data from Romano MR, Montericcio A, Montalbano C, et al. Facing COVID-19 in ophthalmology department. Curr Eye Res. 2020;45(6):653-658. doi:10.1080/ $02713683.2020 .1752737 .^{10}$

questions prior to entering the waiting room comprise one of the initial steps for all patients, as shown in Figure 5. Additionally, essential procedures, including the temperature measurements, are conducted at the main hospital entrance. Social distancing and minimising contact with the healthcare assistants in elective appointments and surgeries were performed. Appropriate sanitation procedures were undertaken to keep the environment safe, including PPE. In addition to some behavioural changes, a breath shield or slitlamb barriers were required, including 1) minimising the patient time spent at the clinic, and 2) replacing all examination contact equipment with non-contact equipment.

A three-level hierarchy developed by various infection control measures based on risk management was introduced by Lai et $\mathrm{al}^{11}$ at the ophthalmology department in Hong Kong (see Figure 6). The first level was the administrative control, which was achieved by rescheduling the appointments and setting up a triage system that postpones appointments for patients identified with COVID-19. Moreover, micro-aerosol generating procedures and nasal endoscopy were stringently avoided, and all elective clinical activities and services were suspended. The second level was environmental control to prevent virus transmission via the instalment of plastic shields on slit-lamps, the disinfection of clinical equipment, and personal temperature measurements, which was advised to all healthcare workers. Finally, the third level was the proper use of PPE.

Each proposed protocol illustrated some strengths and shortfalls in practice as demonstrated in Table 1. 


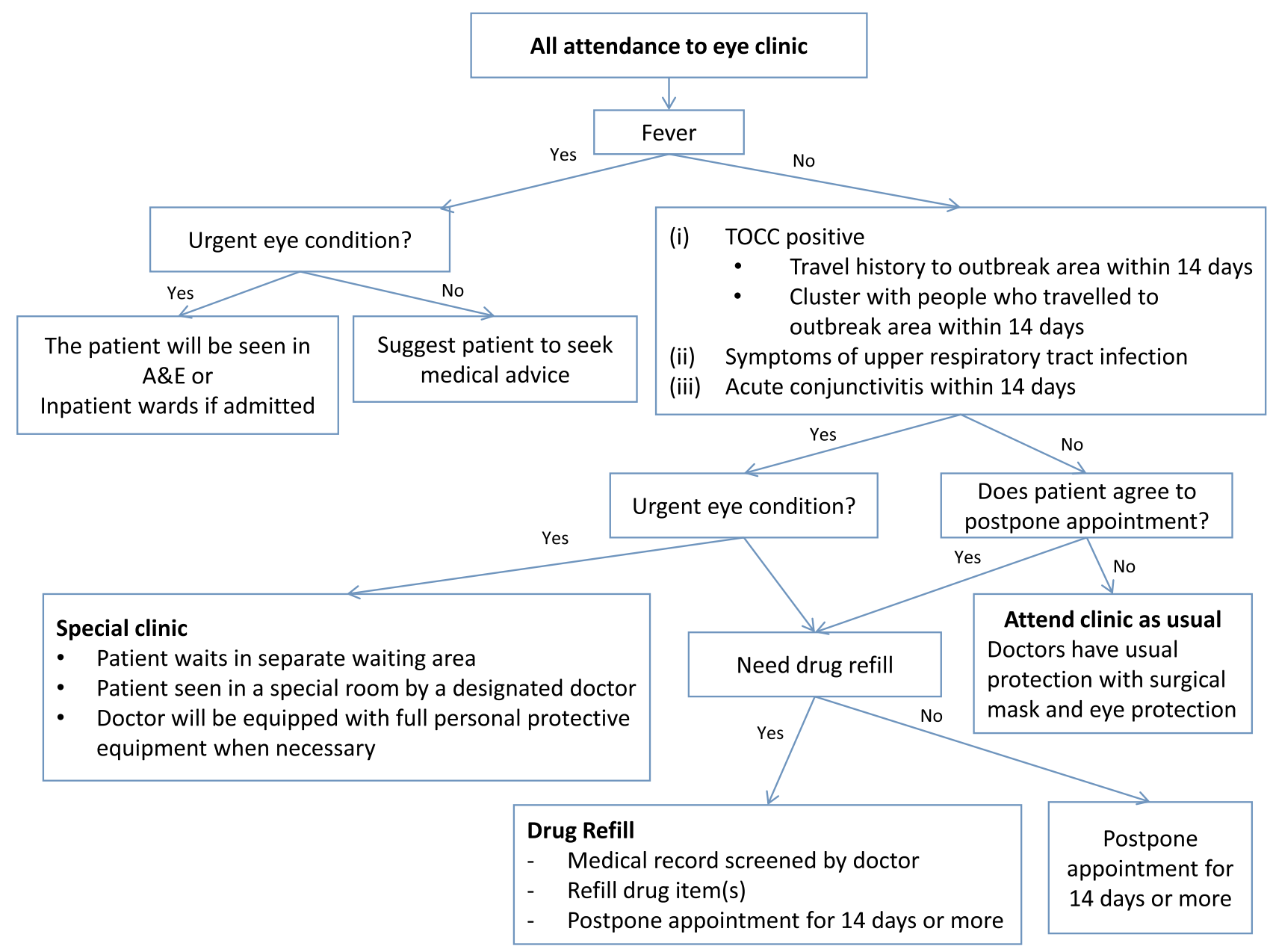

Figure 6 Patient triage in ophthalmology outpatient clinic.

Abbreviations: A\&E, Accident and Emergency Department; TOCC, travel, occupation, contact, and clustering.

Note: Reprinted by permission from Springer Nature Customer Service Centre GmbH: Springer Nature, Graefe's Archive for Clinical and Experimental Ophthalmology, Stepping up infection control measures in ophthalmology during the novel coronavirus outbreak: an experience from Hong Kong, Lai TH, Tang EW, Chau SK, Fung KS, Li KK. Copyright 2020."

Moreover, Table 2 illustrates several protective measures adopted for the aforementioned six ophthalmic practices.

Three clinical protocols have involved virtual clinics; however, no one has considered the AI tools (one approved by the US FDA) ${ }^{12}$ to diagnose/screen at the hospital or the patients' home. The AI applications are non-invasive and assistant, particularly for the follow-up patients (retina and glaucoma). Since the patients' history is recorded for the follow-up appointments, these tools will identify whether there is a need for clinical set examination or not.

Singapore's clinical protocol is the most appropriate among the others for permanent situation. However, the virtual tools and AI applications will add more value to its practice.

\section{Discussion}

Several studies ${ }^{13,14}$ reported positive conjunctival swabs with the presence of conjunctivitis. Thus, there is a possibility of transmission of SARS-CoV-2 through the contact with the ocular surface. ${ }^{15}$ The American Academy of Ophthalmology has addressed temporary advice for ophthalmic practices to maintain open clinics during the pandemic. ${ }^{16}$ Additionally, several protection protocols published to serve the ophthalmology clinic workers and visitors. ${ }^{5,17}$

Furthermore, according to Wong, ${ }^{18}$ COVID-19 has significantly impacted operating the ophthalmology departments in the Academic Medical Centers (AMCs). Hence, the strategic and operational missions of the AMCs and clinical practice are required to institute changes. These changes include: 
Table I The Strengths and Shortfalls of the Six Introduced Protocols

\begin{tabular}{|c|c|c|}
\hline Protocol & Strengths & Shortfalls \\
\hline $\mid$ |srael ${ }^{6}$ & $\begin{array}{l}\text { - Ocular clinical care for the suspected COVID-19 patients (only high tem- } \\
\text { perature }>38^{\circ} \mathrm{C} \text { ) } \\
\text { - Restricted in clinic procedures } \\
\text { - Telemedicine technology }\end{array}$ & $\begin{array}{l}\text { - Temporary protocol } \\
\text { - Limited screening measurements } \\
\text { - No Artificial intelligence (AI) } \\
\text { - No advance diagnostic, inpatients and surgery } \\
\text { - Nrocedures protocols } \\
\text { - No staff feedback }\end{array}$ \\
\hline Singapore ${ }^{7}$ & $\begin{array}{l}\text { - Partially permanent protocol } \\
\text { - Restricted screening to identify the COVID-19 cases for the patients and } \\
\text { their accompanying } \\
\text { - Restricted in clinic procedures } \\
\text { - Outpatients and inpatients protocols } \\
\text { - Ocular clinical care for highly suspected COVID-19 patients } \\
\text { - Staff feedback }\end{array}$ & - No technology (Al or Telemedicine) \\
\hline $\begin{array}{l}\text { Not } \\
\text { specified }^{8}\end{array}$ & $\begin{array}{l}\text { - Ocular emergency protocol } \\
\text { - Restricted in clinic procedures } \\
\text { - Ocular clinical care for highly suspected COVID-19 patients }\end{array}$ & $\begin{array}{l}\text { - Temporary protocol } \\
\text { - No technology (Al or Telemedicine) } \\
\text { - No advance diagnostic, inpatients and surgery } \\
\text { - Nocedures protocols } \\
\text { - No staff feedback }\end{array}$ \\
\hline $\operatorname{Iran}^{9}$ & $\begin{array}{l}\text { - Ocular emergency protocol } \\
\text { - Restricted in clinic procedures } \\
\text { - Telemedicine technology }\end{array}$ & $\begin{array}{l}\text { - Temporary protocol } \\
\text { - No Artificial intelligence (AI) } \\
\text { - No advance diagnostic, inpatients and surgery } \\
\text { - Nrocedures protocols } \\
\text { - No ocular care for suspected COVID-I9 } \\
\text { - No staff feedback } \\
\text { - Postpone non-urgent cases }\end{array}$ \\
\hline Italy ${ }^{10}$ & $\begin{array}{l}\text { - Restricted in clinic procedures } \\
\text { - Telemedicine technology } \\
\text { - Ocular clinical care for tested COVID-19 patients } \\
\text { for negative COVID-19 patients) }\end{array}$ & $\begin{array}{l}\text { - Temporary protocol } \\
\text { - No Artificial intelligence (AI) } \\
\text { - No immediate ocular care for suspected } \\
\text { COVID-I9 patients } \\
\text { - No advance diagnostic, inpatients and surgery } \\
\text { procedures protocols } \\
\text { - No staff feedback }\end{array}$ \\
\hline Hong Kong'I & $\begin{array}{l}\text { - Restricted screening to identify the COVID-19 cases for the patients } \\
\text { - Restricted in clinic procedures } \\
\text { - Ocular emergency protocol } \\
\text { - Ocular non-emergency protocol }\end{array}$ & $\begin{array}{l}\text { - Temporary protocol } \\
\text { - Long screening process } \\
\text { - No technology (Al or Telemedicine) } \\
\text { - No advance diagnostic, inpatients and surgery } \\
\text { procedures protocols } \\
\text { - No staff feedback }\end{array}$ \\
\hline
\end{tabular}

Patient care by developing virtual clinics and telemedicine programmes for screening, monitoring cases, and scheduling appointments in a safe environment outside hospitals. Furthermore, most of the ophthalmology surgery cases are conducted in the main operating room, while the ambulatory surgery centres are closed. Hence, increasing these protocols' precision is necessary to ensure a healthy and safe environment in operation rooms.

Didactic teaching programmes by converting all physical meetings into online platforms.

Clinical teaching programmes, where a group of graduating general physicians will have no ophthalmology training. Hence, AMCs will have to include 


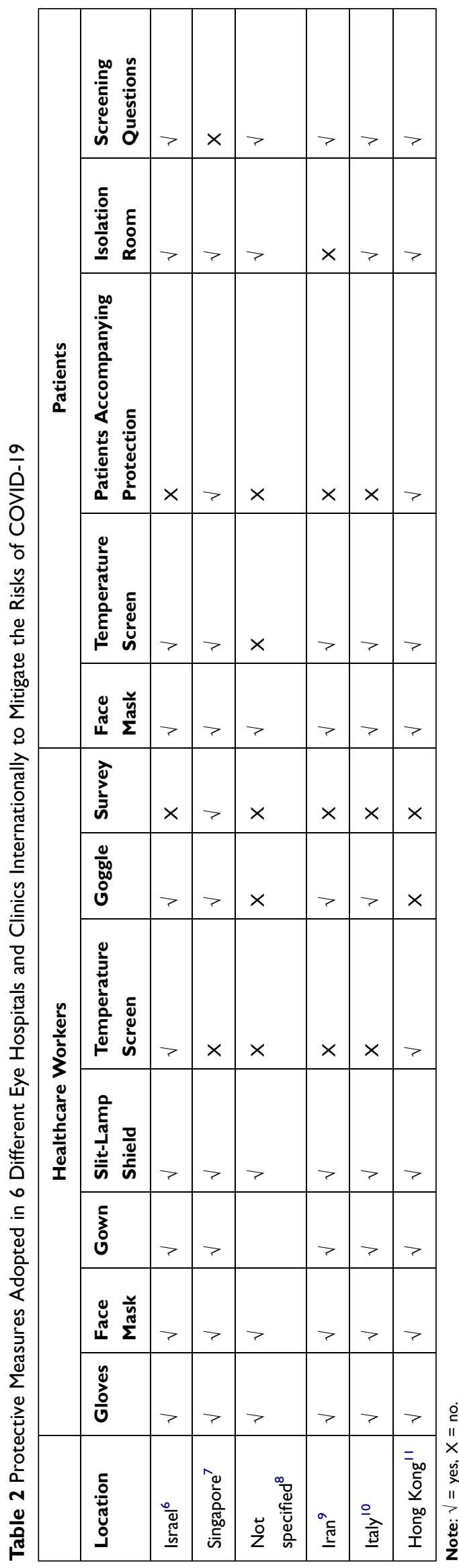

diverse fields, including telemedicine, virtual reality training, and data science.

Clinical research, where only essential research is permitted. However, novel data acquisition, telemedicine, remote monitoring, and online questionnaires will be needed to conduct clinical research.

Basic research, where only certain activities will be permitted in the labs. Therefore, some modifications are likely to be instituted in the basic research protocols.

Ferrara et $\mathrm{al}^{19}$ have distributed an online survey to ophthalmology trainees in 32 different countries to assess the new experience in the ophthalmology training during COVID-19. The results showed an agreement regarding the valuable role of a) web-based case presentations for clinical training, b) web-based discussions of edited surgical videos, and c) simulation-based practice in surgical training.

Lin et $\mathrm{al}^{20}$ stated that the cataract surgery workload would face a $50 \%$ increase post-COVID-19. Thus, they proposed integrated practice units (IPU) with principles of valuebased healthcare. The IPU is used to develop a comprehensive patient-centred care pathway. This is done by combining a streaming system capable of quickly adapting to the changing environment with broader telemedicine applications, such as the Virtual-Plus model and the redesigned patient surgery decision-making aides (PSDMA). On the other hand, the EyeSi simulator has been introduced for the trainees to maintain and practice their skills.

According to Jayaram et al, ${ }^{21} 30,000$ glaucoma outpatients attendance of 120,000 patients have anticipated to be deferred during the pandemic within the glaucoma service across the Moorfields Network. Thus, a "three-tier" approach of consultant-led care has been established and is as follows: a) consultant-led face-to-face clinics, b) optometrist-led clinics, and c) technician-led "virtual" clinics for those patients who are at the lowest risk. Triaging the patients upon their COVID-19 and mediumterm glaucoma risk; thus, the urgent cases have been seen in a hospital setting. Husain et $\mathrm{al}^{22}$ indicated that combining offline and online medical care is essential to manage glaucoma patients. The online examination is conducted for primary consultation, and then the patients who need more investigation or surgical procedures should have inperson management. Additionally, digital technologies 
such as smartphone applications and artificial intelligence represent an avenue to transform glaucoma management.

\section{Conclusion}

The majority of the aforementioned protocols have many similarities in their procedures, especially in postponing elective appointments and surgeries and performing the PPE. However, slight differences still exist. These protocols were instituted during the early onset of COVID-19. Thus, the hospitals' preparations for such a pandemic were not yet complete. The sustainability of operating clinics, whether inpatient or outpatient, is the primary goal in overcoming the issues that accompany this pandemic or any potential future pandemic. Hence, the introduction of solutions is necessary.

In addition to the principal procedures that were previously advised, additional procedures are recommended in order to maintain the clinics' sustainability, through 1) installation of a permanent infection control unit at the hospital entrance to ensure that all visitors have passed requirements to enter the hospital; otherwise, they are referred to a dedicated clinic (preparing an outside clinic dedicated for COVID-19 patients or suspect cases) in case of any related symptoms, and 2) extension of the working hours and days in the ophthalmic clinic for inpatient or outpatient services, to serve the regular number of patients as well as to release the work pressure for the staff. For example, instead of eight hours a day and five days a week, 12 hours a day and six days a week could be a solution to; a) have more time spent in sanitising the clinic, b) reduction of the number of patients in the waiting area, and c) reduction the HCWs exposure to COVID-19.

Imaging services are essential diagnostic tools and required by most ophthalmology subspecialties, and the patients are visiting the imaging places very frequently. Hence, a crucial consideration is required for preventing the COVID-19 between the patients and HCWs. Imaging devices should be placed in separate rooms. Otherwise, the fundus images can be taken by smartphone camera or portable fundus camera. Then, one can use the developed algorithms to measure various retinal diseases such as diabetic retinopathy level and progression and glaucoma represented by the cup-todisc ratio.

Additionally, more attention to telemedicine innovations is required by innovators to use smartphone tools, video calls, and portable ophthalmic equipment to make eye clinics more practical for providing sustainability in healthcare services.

\section{Disclosure}

The author reports no conflicts of interest in this work.

\section{References}

1. PAHO. COVID-19 has infected some 570,000 health workers and killed 2500 in the Americas; September, 2020. Available from: https://www.paho.org/en/news/2-9-2020-covid-19-has-infected-some -570000-health-workers-and-killed-2500-americas-paho. Accessed January, 2021.

2. Bandyopadhyay $\mathrm{S}$, Baticulon RE, Kadhum $\mathrm{M}$, et al. Infection and mortality of healthcare workers worldwide from COVID-19: a systematic review. BMJ Glob Health. 2020;5(12):e003097. doi:10.1136/bmjgh-2020-003097

3. Nguyen LH, Drew DA, Graham MS, et al. Risk of COVID-19 among front-line health-care workers and the general community: a prospective cohort study. Lancet Public Health. 2020;5(9):e475e483. doi:10.1016/S2468-2667(20)30164-X

4. Greenberg N. Mental health of health-care workers in the COVID-19 era. Nat Rev Nephrol. 2020:1-2. doi:10.1038/s41581019-0226-4

5. Li JPO, Shantha J, Wong TY, et al. Preparedness among ophthalmologists: during and beyond the COVID-19 pandemic. Ophthalmology. 2020;127(5):569-572. doi:10.1016/j.ophtha.2020.03.037

6. Safadi K, Kruger JM, Chowers I, et al. Ophthalmology practice during the COVID-19 pandemic. BMJ Open Ophthalmol. 2020;5 (1):e000487. doi:10.1136/bmjophth-2020-000487

7. Lim LW, Yip LW, Tay HW, Ang XL, Lee LK. Sustainable practice of ophthalmology during COVID-19: challenges and solutions. Graefes Arch Clin Exp Ophthalmol. 2020;258(7):1427-1436. doi:10.1007/ s00417-020-04682-z

8. Sadhu S, Agrawal R, Pyare R, et al. COVID-19: limiting the risks for eye care professionals. Ocul Immunol Inflamm. 2020;28(5):714-720. doi:10.1080/09273948.2020.1755442

9. Gharebaghi R, Desuatels J, Moshirfar M, Parvizi M, Daryabari SH, Heidary F. COVID-19 preliminary clinical guidelines for ophthalmology practices. Med Hypothesis Discov Innov Ophthalmol. 2020;8 (2): 149 .

10. Romano MR, Montericcio A, Montalbano C, et al. Facing COVID-19 in ophthalmology department. Curr Eye Res. 2020;45(6):653-658. doi:10.1080/02713683.2020.1752737

11. Lai TH, Tang EW, Chau SK, Fung KS, Li KK. Stepping up infection control measures in ophthalmology during the novel coronavirus outbreak: an experience from Hong Kong. Graefes Arch Clin Exp Ophthalmol. 2020;258(5):1049-1055. doi:10.1007/s00417-02004641-8

12. FDA; 2018. Available from: https://www.fda.gov/news-events/pressannouncements/fda-permits-marketing-artificial-intelligence-baseddevice-detect-certain-diabetes-related-eye. Accessed January, 2021.

13. Cheema M, Aghazadeh H, Nazarali S, et al. Keratoconjunctivitis as the initial medical presentation of the novel coronavirus disease 2019 (COVID-19). Can J Ophthalmol. 2020;55(4):e125-e129. doi:10.1016/j.jcjo.2020.03.003

14. Colavita F, Lapa D, Carletti F, et al. SARS-CoV-2 isolation from ocular secretions of a patient with COVID-19 in Italy with prolonged viral RNA detection. Ann Intern Med. 2020;173(3):242-243. doi:10.7326/M20-1176 
15. Sitaula RK, Khatri A, Janani MK, et al. Unfolding COVID-19: lessons-in-learning in ophthalmology. Clin Ophthalmol. 2020;14:2807. doi:10.2147/OPTH.S259857

16. AAO. important coronavirus updates for ophthalmologists; 2020. Available from: https://www.aao.org/headline/alert-importantcoronavirus-context. Accessed January, 2021.

17. Limbu B, Khatri A, Joshi P, et al. COVID-19 preferred practice pattern for eye care professional-An initiative by Nepal Ophthalmic Society. Nepal J Ophthalmol. 2020;12(2):176-190. doi:10.3126/nepjoph.v12i2.31835

18. Wong TY, Bandello F. Academic Ophthalmology during and after the COVID-19 Pandemic. Ophthalmology. 2020;127(8):e51-e52. doi:10.1016/j.ophtha.2020.04.029
19. Ferrara M, Romano V, Steel DH, et al. Reshaping ophthalmology training after COVID-19 pandemic. Eye. 2020;34(11):2089-2097. doi:10.1038/s41433-020-1061-3

20. Lin PF, Naveed H, Eleftheriadou M, Purbrick R, Ghanavati MZ, Liu C. Cataract service redesign in the post-COVID-19 era. $\mathrm{Br}$ J Ophthalmol. 2020. doi:10.1136/bjophthalmol-2020-316917

21. Jayaram H, Strouthidis NG, Gazzard G. The COVID-19 pandemic will redefine the future delivery of glaucoma care. Eye. 2020:1-3.

22. Husain R, Zhang X, Aung T. Challenges and lessons for managing glaucoma during COVID-19 pandemic: perspectives from Asia. Ophthalmology. 2020;127(9):e63-e64. doi:10.1016/j. ophtha.2020.05.042
Clinical Ophthalmology

\section{Publish your work in this journal}

Clinical Ophthalmology is an international, peer-reviewed journal covering all subspecialties within ophthalmology. Key topics include: Optometry; Visual science; Pharmacology and drug therapy in eye diseases; Basic Sciences; Primary and Secondary eye care; Patient Safety and Quality of Care Improvements. This journal is indexed on PubMed

Submit your manuscript here: https://www.dovepress.com/clinical-ophthalmology-journal
Dovepress

Central and CAS, and is the official journal of The Society of Clinical Ophthalmology (SCO). The manuscript management system is completely online and includes a very quick and fair peer-review system, which is all easy to use. Visit http://www.dovepress.com/ testimonials.php to read real quotes from published authors. 